Canadian Journal of Higher Education Revue canadienne d'enseignement supérieur

Volume 46, No. 4, 2016, pages $60-75$

\title{
A Faculty-Based Mentorship Circle: Positioning New Faculty for Success
}

Janice Waddell, Jennifer Martin, Jasna K. Schwind, and Jennifer L. Lapum Ryerson University

\begin{abstract}
Multiple and competing priorities within a dynamic and changing academic environment can pose significant challenges for new faculty. Mentorship has been identified as an important strategy to help socialize new faculty to their roles and the expectations of the academic environment. It also helps them learn new skills that will position them to be successful in their academic career. In this article, the authors report on the implementation and evaluation of a mentorship circle initiative aimed at supporting new faculty in the first two years of their academic appointment. Participants reported that the mentorship circle provided them with a culture of support, a sense of belonging, and a safe space to discuss concerns and learn strategies from both mentors and fellow mentees as they adjusted to their new position. The interdisciplinary nature of the mentorship circle further facilitated faculty members' capacity to navigate their role as new faculty and foster colleagueship.
\end{abstract}

\section{Résumé}

Dans un milieu universitaire dynamique et évolutif, des priorités diverses et contradictoires peuvent représenter des défis importants pour les nouveaux membres du corps professoral. Le mentorat a été identifié comme une stratégie importante qui aide les nouveaux membres du corps professoral à socialiser dans le cadre de leurs fonctions et à répondre aux attentes du milieu universitaire. Le mentorat les aide aussi à acquérir de nouvelles compétences qui les prépareront à réussir leur carrière universitaire. Dans cet article, les auteurs font état de la mise en œuvre et de l'évaluation d'un cercle de mentorat visant à soutenir les nouveaux membres du corps professoral au cours de leurs deux premières années en fonction. Les participants ont signalé que le cercle de mentorat leur avait offert une culture de soutien, un sentiment d'appartenance et un espace sûr pour discuter de leurs préoccupations et acquérir, grâce au contact des mentors et d'autres collègues du cercle, des 
stratégies pour s'adapter à leur nouveau rôle. La nature interdisciplinaire du cercle de mentorat a facilité davantage la capacité des membres du corps enseignant à mener leur rôle et à cultiver des relations collégiales.

Mentorship is most often described as a guiding relationship (Zeind et al., 2005) in which a more experienced person, the mentor, assumes a supportive role by overseeing and encouraging reflection and learning with a less-experienced person, the mentee (Gazza \& Shellenberger, 2005). The goal of a mentorship relationship is to provide an environment of support and to advise and coach an individual within the context of the culture and expectations of the organization (Angelique, Kyle, \& Taylor, 2002). In this article, we discuss the implementation and the evaluation of a mentorship circle for university faculty new to the institution. We first provide an overview of the importance and types of mentorship. Next, we review background to the structure and process of our mentorship initiative. Last, we share the evaluative feedback and lessons learned.

\section{Importance of Mentorship for New Faculty}

The academic landscape is far different today than it was a few decades ago when formal mentoring programs were introduced to higher education (Altbach, 2000; Belar, 1998). There has been a rise in part-time and nontenure-track faculty positions, resulting in altered systems of tenure and promotion. Moreover, faculty members across the career continuum are under increased pressure to develop a strong research program, to publish, and to teach more courses, all the while adopting new technologies and pedagogies. These multiple and competing priorities, within the context of a dynamic academic environment, can be a significant challenge for new faculty who may have minimal guidance to navigate such expectations.

New faculty express multiple needs within their first year, including the desire to connect with colleagues, receive help navigating the political structure of the organization, and obtain support for efficient functioning (Gazza \& Shellenberger, 2005; White, Brannan, \& Wilson, 2010). Siler and Kleiner (2001) suggest that faculty newcomers, in addition to balancing priorities within an unfamiliar environment, may have an additional disadvantage due to the lack of preparation in their graduate program on how to effectively function in an academic role. They posit that mentorship is important to help socialize new faculty to their roles and the expectations of the academic environment, and to help them learn new skills that will position them to be successful in their academic career.

There are many implicit rules within the academic setting, which are shaped by the university's political landscape. Mentorship may help new faculty understand existing hierarchies and provide insight regarding how best to work within the system (Gazza \& Shellenberger, 2005). Many new faculty describe time management as a significant challenge that contributes considerably to the stress in their new role (Oosthuizen, Mckay, \& Sharpe, 2005). Learning new strategies within the mentorship relationship help new faculty overcome frustration and develop practical skills, which are key to being an efficient educator (White et al., 2010).

Mentorship is also proposed as a means to support the psychosocial needs of new faculty (Angelique et al., 2002). New faculty members express the importance of feeling included while developing new connections, and they highlight the importance of being supported by an institution that values their well-being (White et al., 2010). Furthermore, 
White and colleagues suggest that psychosocial support can diminish the stress that new faculty experience in the first few years of their academic career. To meet these needs, it behooves academic institutions to provide new faculty with both instrumental and emotional support. Oosthuizen et al. (2005) propose that problems encountered by academics in their early years can, with the support and guidance from more experienced faculty, be alleviated by quickly establishing a productive and supportive network of colleagues and learning time-management strategies to balance work demands (White et al., 2010). To address psychosocial needs, institutions can provide an inclusive environment, a comprehensive orientation, and the means to network and connect with other faculty (Gazza \& Shellenberger, 2005; Oosthuizen et al., 2005).

\section{Types of Mentorship: Strengths and Challenges}

Traditional mentorship models are common in the academic environment (Angelique et al., 2002). Traditional mentoring describes a one-to-one, uni-directional, asymmetrical relationship in which a junior, or a less experienced person, is paired with a more experienced person who provides guidance and support (Blackwell, 1989). There are limitations to the traditional didactic model and the literature suggests a need for innovative, more effective models that address the needs of faculty working within the contexts of contemporary academic institutions (Darwin \& Palmer, 2009). While the traditional mentorship model provides faculty with support and coaching with a more experienced colleague, this relationship can propagate a dynamic of power. The mentor is generally the one in control of the mentor-mentee relationship, and as such, the power dynamic can have the potential to be exploitative (Angelique et al., 2002). This form of mentorship also limits new faculty to a single point of view. Darwin and Palmer (2009) suggest that to be successful in today's academic environment, one must have access to various mentors, perspectives and insights.

To address the potential drawbacks of a traditional dyadic mentorship relationship, alternative forms of mentoring have emerged. Peer mentoring brings faculty together to share information and can occur within dyads where one peer has slightly more experience than the other member of the dyad (Beane-Katner, 2014). By pairing individuals with those of the same experience, rank, and hierarchal level within the institution, new faculty have an opportunity to meet others in the same situation as themselves, thereby fostering a sense of inclusiveness and well-being (Angelique et al., 2002). Peer mentorship has the potential to create a more equitable environment, and drawing on commonalities, participants have the opportunity to be more empathetic (Angelique et al., 2002). However, Angelique and colleagues caution that a purely peer mentorship model has the potential for competitiveness amongst peers. A variant of a dyadic peer mentorship approach is mutual mentoring, a format that provides new faculty members with the opportunity to mentor one another directly (Beane-Katner, 2014).

Beane-Katner (2014) suggests that both peer and mutual mentorship can occur in groups where faculty members with similar characteristics and experiences establish networks that serve to build a sense of community and shared understanding of the faculty role. This author also believes that peer and mutual mentorship groups can benefit from the input and the perspectives of more experienced faculty members. Darwin and Palmer (2009) describe a mentorship circle model that draws on strengths from the above formats. It is an innovative model that fosters mentorship relationships and typically involves more experienced faculty facilitator(s) serving in the role of mentor with a group of new faculty 
peers. A mentorship circle approach, based on the premise that individuals learn in relationships, offers flexibility, diversity, and knowledge creation by exposing the mentees to various perspectives, including those of the mentees themselves (Darwin \& Palmer, 2009).

\section{Our Mentorship Initiative}

As a group of four faculty mentors within one faculty in an urban university, we collaborated to construct a mentorship initiative aimed at supporting new faculty in the first two years of their academic role. At the inception of the mentorship circle initiative in fall 2012, two nursing faculty mentors were in the process of preparing their tenure applications, a third child and youth care faculty mentor was in the second year of a tenure-track appointment, and the fourth mentor was a long-standing faculty member also serving in the role of associate dean of the faculty.

The impetus for this initiative was based on the extant literature that highlights the positive role mentorship could play in the experience of new faculty members and the desire to support newcomers during their transition to our academic institution. After reviewing the mentorship literature we decided that the mentorship circle model as described by Darwin and Palmer (2009) was a good fit within the context of our faculty, which is comprised of nine professional programs.

The design and process of the mentorship circle were guided by findings and recommendations from an evaluative study of three approaches to mentorship described by Darwin and Palmer (2009). They identified the structure and process of mentorship circles that were reported by the study participants to be the most helpful and relevant to their experience as new faculty members. Broadly, they recommended that participation in mentorship circles be voluntary and include interdisciplinary faculty. The following elements highlighted as integral to successful implementation of mentorship circles were that

- discussions be facilitated by faculty members who are a source of information for the members,

- discussions be guided by a concrete list of themes generated by the members,

- notes be sent out to group members after each session,

- the mentorship circle be one of many resources/supports available to faculty members (Darwin \& Palmer, 2009),

With these guidelines in mind, we expanded our mentorship circle to include recently hired faculty from across the schools. Faculty members in the first two years of employment at the university were sent a written invitation to participate in the mentorship circle. Seventy-five percent of those contacted accepted the invitation and participated in the regularly scheduled meetings over the course of the academic year. There were nine mentees from the Faculty of Community Services, specifically from the schools of Nursing, Early Childhood Studies, Nutrition, Disability Studies, Occupational and Public Health, and Social Work.

Once meetings began, if a mentee was unable to attend a meeting, all materials from the meeting were forwarded to the individual with an invitation to contact any of the mentors with questions or to discuss what transpired during the discussion. To reduce the time and energy necessary to bring mentees together, mentorship circle meetings were coordinated by a staff member in the dean's office. This individual also arranged for food at each meeting, and created and distributed resource material folders, specific to each of the meeting discussion themes. 
The mentorship circle met five times over the course of the academic year. During the first two meetings the mentees shared expectations for their involvement in the mentorship circle, developed group norms, and generated a number of thematic topics they wished to discuss. Examples of themes included developing one's program of research, creating a teaching philosophy and dossier, preparing one's annual report, and building a tenure application. In addition to the themed discussion, it was decided that a portion of each meeting would be dedicated to a general check-in to allow members to share and consult with one another about their day-to-day experiences in their new role. Mentees were encouraged to contact one another and/or the mentors between meetings, as needed. In addition to participation in the mentorship circle, mentees had access to a facultywide professional development program, Positioning for Success in Academia (PFS). The PFS program includes interactive workshops focused on issues or topics of interest for faculty members in their academic role. Mentees were referred to specific PFS workshops responsive to specific informational or development needs expressed in the mentorship circle meetings. Members often brought information from the PFS workshops to the circle for the purposes of sharing and further discussion.

\section{Project Evaluation}

A graduate project assistant emailed all the mentees an invitation to a focus group to evaluate the mentorship initiative. Participation was voluntary and their decision was confidential (the mentors were not made aware of who decided to participate or not participate). The project assistant also conducted a focus group with us, the mentors. Although our experiences are not the main focus of this article, we incorporate some of these perspectives in the discussion section related to our lessons learned.

Focus group analysis was conducted by the project assistant and the mentors, and the following provides an overview of the perspectives of the mentees who participated in the focus group discussions. Five themes emerged from this analysis: (1) structure of sessions, (2) culture of support, (3) gaining insight into the faculty member's role, (4) investing in self and others, and (5) the ripple effect. (Table 1)

Table 1.

Overview of the Main Themes

\begin{tabular}{ll}
\hline Themes & Description \\
\hline Structure of sessions & $\begin{array}{l}\text { Blend of predetermined and emergent structure of sessions that } \\
\text { are flexible and responsive to mentees' needs. } \\
\text { Sufe and supportive space to build collegial relationships and } \\
\text { foster a sense of community. Mentees appreciated the space } \\
\text { and time to collaborate and share experiences. }\end{array}$ \\
$\begin{array}{ll}\text { Gaining insight into the fac- } \\
\text { ulty member's role }\end{array}$ & $\begin{array}{l}\text { Strategically managed integration into the academic commu- } \\
\text { nity as a result of learning the role expectations. }\end{array}$ \\
Investing in self and others & $\begin{array}{l}\text { Investment in the well-being of mentees through discussion of } \\
\text { healthy work-life balance }\end{array}$ \\
The ripple effect & $\begin{array}{l}\text { Catalyst to changing the culture within the university by pro- } \\
\text { moting collegial support; perceived by members to promote } \\
\text { faculty recruitment and retention. }\end{array}$ \\
\hline
\end{tabular}




\section{Structure of Sessions}

The blend of formal and informal formats to structure the mentorship sessions elicited mixed, but mostly positive, reactions, Knowing the topics ahead of time helped some mentees prioritize their work schedule and attend sessions based upon the relevance of the topics. When describing the structure of the mentorship circle, one mentee expected the sessions would be more informal, resembling a support group:

I thought it would be less structured. I'm not sure if I mean that as a critique at all. Just, I thought it would be a little bit more support group-ish and maybe a little less

"Pick a topic, discuss that topic." I'm not sure if I would have actually liked that better.

The same mentee also suggested that the number of mentees may have influenced how sessions were organized, and why the sense of a support group did not materialize: "I think, maybe, the reason it didn't go in that direction is the group was quite large. Perhaps it would have had a different vibe if there were [only] five of us."

While some expected the mentorship circle to be more of an informal support group, other mentees liked the idea of having predetermined themes to structure the meetings:

The structure of it, I thought maybe it was more of a "let's come together and what are your concerns, what do you need to know about?" But on the opposite side of that, I did like the structure and that there was a topic.

The combination of flexible discussions and scheduled themes provided a framework within which all mentees could engage and support each other.

Mentees' comments also demonstrated how mentors were responsive to the needs of group members. At the end of each meeting, topics were determined by mentees based on what was deemed to be relevant and beneficial to them. As indicated by one mentee, "It was nice to be asked what topics might be useful as opposed to a list of topics that are kind of imposed. That was helpful. It seemed like there was an authenticity to the mentorship because of that." Another mentee echoed this appreciation:

There wasn't a set agenda, even though there was a topic; it wasn't like, "We need to cover these things." I did find that, and appreciated that, because that's whatthose who were there for the mentorship-that's what they needed at that time.

The emergent nature of the sessions was perceived by mentees to be effective and authentic. Another mentee remarked on the mentors' flexibility in guiding the group discussions to suit members' interests:

I noticed also in the mentorship circle, if it [the discussions] took another road, it wasn't stopped either. We went down that road. Sometimes we did go off on tangents, but it was a good tangent. That's what our needs were at that time.

The responsiveness to mentees' needs and thus the emergent nature of topics was identified as a strength of the mentorship circle sessions.

Mentees expressed an unmet need and interest in maintaining connections by having follow-up sessions with mentees among themselves outside the organized meetings. A mentee elaborated: 
Follow-up is on-going communication via email. Not just when the meeting [will take place], not just to schedule the meeting, but I wished it was . . . a discussion group where we talk during the process. If somebody comes up with something or learns something, they'll share it with the group. After all, it's about mentoring. It shouldn't just be, you know, waiting till the next month.

As this excerpt demonstrates, mentees wanted a continuous, ongoing form of mentorship. The above excerpt also suggests that mentees could better engage in the mentorship process by sharing knowledge with one another and building relationships outside the scheduled meetings. The ongoing communication via email was an aspect of the mentorship circle that mentees perceived as valuable, a way to build on previously discussed topics and keep the momentum going. This connection was deemed particularly important if a mentee missed a meeting, as articulated by the following mentee:

There was one [session] I couldn't go to and I was trying to get information about what happened there. It's kind of hard because there's that [feeling], once you leave the mentorship circle, it's almost like a disconnect, "What happened there? I wish I had that information." It's the follow-up-if you don't attend for whatever reason, that you're still able to get information. I know sometimes there're booklets being given out, resources. For me, anyhow, it would be the follow-up [that's important].

Although the material resources were deemed helpful, it was evident that an opening for dialogue between the monthly sessions was desired.

Mentees indicated that integrated structure and organization of the mentorship circle was important and helped to set norms and clarify expectations. Specifically, they indicated the importance of norms and expectations regarding trust and privacy amid awareness of power differences between mentees:

We have to acknowledge the power differential, and the structure of the academy is such that our colleagues are the ones evaluating us at the end of the day. I think we have to be aware of that inherent power differential that new faculty members are subject to. I think in recognition of that, in order for it to be a truly authentic mentorship relationship, there has to be that safety right up front. I think it was certainly there after the first meeting - the last thing you want is having a mentor in another department who's then going to turn around and talk to your chair or to your colleague about what you're sharing, especially if you didn't want it to be shared.

As richly reflected in this excerpt, creating a safe space was acknowledged as vital for optimal mentorship in-group sessions.

Establishing a clear definition of mentorship and clarification of the mentoring relationship considered important by mentees because people may define the term and understand the roles of mentor and mentee in various ways. The potential for fostering a mentoring relationship that develops over time within the context of a larger mentorship circle was described as being preferable to a traditional, assigned mentor in a one-to-one mentoring role:

When I think about the people that have mentored me throughout my career, none of them have been assigned to me. I don't know if I would want someone assigned 
to me and say, "This is going to be your mentor"; because I think it works out ofonce again-the relationship. But I think going into this group ... I went in with, "Wow, there might be someone there or other people that I can really look up to."

As reflected in this excerpt, optimal mentoring occurs when, through relationship building, an organic connection develops.

\section{Culture of Support}

Participation in the mentorship circle allowed mentees to establish and maintain supportive and collegial relationships with others, which fostered a sense of community and collaboration. Mentorship sessions were perceived by the mentees to be a safe space to build relationships with colleagues from different schools who may be experiencing similar challenges in adjusting to a new academic role. The fundamental importance of feeling safe underpinned several themes and was identified by mentees as a vital component of the mentorship circle. As the following mentee observed:

I think that the safety piece was key and I personally felt it was established. It was more about sharing and people having a chance to share.... I didn't see any silenced voices around the table, and that helped to motivate my continued engagement.

This quote demonstrates how a sense of safety promotes inclusion, rapport, and support for all mentees.

The mentorship circle promoted a learning environment in which mentees shared their experiences, listened carefully without judgment, asked clarifying questions, and offered thoughtful feedback to each other. Mentees also discussed how participation in the mentorship sessions promoted a broader culture of support and development of interdisciplinary collaboration. These newly formed connections thus offered the opportunity for mentees to learn with and from one another as well as from the mentors. One mentee stated:

What was well appreciated was that there were colleagues from different stages in their career . . . opportunity for fellowship across disciplines, I think, adds value to our work, to our personal development. ... it's beneficial not just for our roles, although ... [it] adds indirectly to our roles, but also it's just good for fellowship. It builds community.

The references to fellowship and community building suggest that a sense of camaraderie developed amongst the mentees.

Some mentees expressed a desire for opportunities to work in smaller, intimate groups of three to four, in order to establish closer professional connections to colleagues. They believed that working in such small groups would strengthen the more personal connections between mentees as well as with the mentors. A mentee commented:

I do agree with what's been said about the importance of maybe smaller groups.... If there's a sub-group of [those] who are in the same year and [you] want to actually go off and do your own thing, just state it, because I think we're going to do it anyway.

After further discussion with mentees, it became apparent that some have informally created their own small community of support. For example, a small group of mentees from 
different schools worked together and supported each other in the preparation of their interim tenure dossiers.

Mentees also noted how participation in the mentorship circle and the opportunities to engage in interdisciplinary dialogue spilled into other aspects of the faculty role, which had an impact on individual values and on interactions with peers and students. Participation in the mentorship circle promoted a sense of belonging to something bigger than one's school-an engagement with the greater university culture. One of the mentees observed, "If I didn't have this mentorship circle, there is no way I would [have met] other people in other [schools], even though we belong to the same [faculty]." As reflected in this quote, the mentorship circle extended mentees' networks and advanced their sense of belonging at a broader institutional level.

\section{Gaining Insight into the Faculty Member's Role}

The mentorship circle provided support and information to facilitate mentees' transition as new faculty members. One mentee referred to how individual needs change over time and how the mentorship circle is beneficial at each stage, leading "up until my tenure." This mentee stated:

I had certain questions in the first year that were answered, and [in] the second year; but now, going into my third year, I still have new questions that arise, "What do I do?” So, I'm still trying to find ... [to] navigate my way through this.

This mentee's comment suggests that the mentorship circle was a resource that assisted her to strategically manage her integration into the academic community. Yet another mentee indicated that the mentorship circle was beneficial in understanding the role expectations from both school and faculty levels:

Having an idea or clarity about what is expected from me, as a new staff [member], at all different levels, is very important. I think the mentorship circle being situated here at the faculty level is important because we have a good sense of what is expected from us at the departmental [school] level and, as well, what's expected on the faculty level.

Although multiple sources of information were available and acknowledged, the mentorship circle was referred to as a credible resource where a wide array of information could help steer group members through their journey as new faculty. One mentee stated:

I can say that there was nowhere [else] that I could go [where] I would've gotten all that information. I probably would have gone to six or seven people with very direct questions in order to get that information.

This mentee referred specifically to a session about the faculty annual report. At our institution, each faculty member is expected to complete an annual report documenting evidence related to their accomplishments in teaching, research, and service; this report has great importance for preparing one's tenure and promotion documents. Another mentee added that "having other people ask questions [such as], 'Well, I have this. Where do I put that?' led me to think, 'Oh, I have the same [issue].' I never even thought about that." Hence, mentees' questions at the circle stimulated extensive dialogue and learning. 
The mentorship circle provided the opportunity for faculty role expectations to be explicated. Two mentees said it helped to "demystify" aspects of the faculty role. The use of demystify suggests that some role expectations are perceived to be obscure, requiring some level of clarification or interpretation. One mentee explained that although "colleagues are a great resource, oftentimes their ideas of what expectations are, [are] based on another collective agreement." This statement reinforces the uncertainty that can surround the dynamic nature of expectations, and the challenge this may pose for new faculty. In referring to role expectations and positioning oneself for tenure, another mentee mentioned that "hearing from other people who have... gone through the process in the mentorship circle was helpful." A third mentee elaborated how the mentorship circle "really succeeded":

When we triangulate all the different pieces of information we received ... you emerge with some vague idea, at least, of becoming slightly more concrete in terms of what the expectations of this job are.

Thus, the mentorship circle was credited as a place to clarify norms and obtain accurate, reliable information: "There's a lot of ... confusion about what the expectations are at the departmental level. ... So, it's nice to actually go someplace where you can count on the information"

Participation in the mentorship circle helped mentees to learn about and understand the variety of expectations across the nine schools within their faculty. Departmental differences were discussed constructively, and mentees benefitted from understanding the diversity, for instance, by contextualizing their priorities in a more political way:

Unpacking some of those disparities was useful.... [I was] shocked at some of the differences, and it gives us . . leverage to go back and say, "No, I need to decline that service commitment," or "I need to be thoughtful about how I select my classes." It gives you a sense of what's normal and what's beyond the pale.

As mentees explored the varied norms, they became more aware and confident about the scope of what was deemed reasonable in terms of role expectations and their own stance.

\section{Investing in Self and Others}

The mentorship circle offered mentees more than strategies to achieve success according to the objective criteria for their academic roles. Mentees spoke about the intangible, hard-to-measure gains from participating in the circle and reflected how the circle was also an investment in the well-being of the mentees, not just a program geared to fostering their ability to meet objective performance standards. The difficulty in measuring how the mentorship circle has benefitted the faculty-at-large was expressed by one of the mentees:

I think when we ask if [the mentorship circle] has increased our service or teaching or SRC [research] capacity, that we are asking the wrong question. . . . It doesn't just change the culture, it changes our individual sense of ourselves in this role . .. how we interact with one another, our students ... I would be very sad to be a 
part of a faculty that only puts forth programs that result in measurable outcomes. ... It's a place that is first and foremost meant to be accountable to its members rather than being an output-based program. This speaks to me as a broader issue of investment ... not only investing in things that are measurable.

Another mentee added: "It helps the faculty in feeling that we all belong underneath this umbrella, and that even though we come from different departments, we all belong to one faculty." In addition to fostering a sense of belonging, the umbrella metaphor suggests a protective capacity that has emerged as a result of the mentorship circle.

\section{The Ripple Effect}

Mentees described the potential of the mentorship circle as a catalyst for changing the culture within the university-at-large by promoting ongoing collegial support:

I feel this [mentorship circle] would contribute to the culture of mentorship if it were to continue year after year because I'd imagine those of us who've benefited from something like this might have already been inclined, or are even more inclined, to actually give back in that same way, whether it's formally as a mentor or informally by contributing to our departmental culture.

The expanding circle of mentorship over time is highlighted in this excerpt, speaking to the ripple effect that can occur throughout the university.

The benefits of the mentorship circle were also perceived by mentees to extend beyond the walls of the faculty and the university, for instance, in promoting faculty recruitment and retention, and serving as a best practice:

I think that the reputation of this faculty is enhanced, because I know I've had conversations with other new faculty members from other universities and when I tell them that I'm in a mentorship group, they're like, "What? How come we don't have that?" And they articulate how much it would help them. I think that if an institution wished to recruit and retain faculty members, mentorship is an important thing. So, absolutely, it's good for [our university's] reputation. It's also good for, you know, us telling our colleagues, "Hey, you know what? You can come in and people will look out for you at the faculty level." I think that's actually helpful in marketing the institution itself to other people.

The mentorship circle was clearly viewed as a form of institutional support for faculty development and success:

Yes, you have to put in the work, but there are other people there who are supporting you through that process. ... When you tell people ... from outside the university ... or even within, there are individuals who didn't have the opportunity to be part of this mentorship circle who say, "You're one of the lucky ones. That was not here when I first started." I think it helps, and it shows that the university is there. Not that you just got your foot in the door, but they're there to help you out throughout the process and that they care. The university cares about how you progress in your profession. 
Throughout these excerpts, the idea of caring and support is evident. The observations of mentees also speak to how this culture of mentorship is viewed favourably outside the university and how it can act as a recruitment and retention strategy for the university.

\section{Discussion: Lessons Learned}

In evaluating this mentorship circle initiative, we found that creating a safe and supportive space for faculty new to the institution was paramount to its success. As the mentorship circle mentees confirmed, the faculty role often involves high expectations in competitive environments (Darwin \& Palmer, 2009; Rice, Sorcinelli, \& Austin, 2000) with multiple competing demands (Chase et al., 2013; Savage, Karp, \& Logue, 2004). As a result, adjusting to a university faculty role can induce a sense of isolation and loneliness (Rice et al., 2000), and vulnerability and anxiety. In designing the mentorship circle, we were conscious of these issues and strove to create a safe harbour where relationships of trust could develop among the mentees and with us, as mentors. As noted by mentees, this sense of safety was essential and was achieved by acknowledging the potential power differentials and by setting group norms early. As mentors, we also recognized that, beyond offering helpful tips, our own personal disclosures about things we wished we had known and things we wished we had done differently as new faculty members fostered trust and allowed mentees to confide their vulnerabilities within the mentorship circle. The resulting rapport was undoubtedly a factor in the mentees' feeling that the institution cared about their overall well-being.

Our evaluation results echo the research findings of Darwin and Palmer (2009) that showed the group mentoring approach embedded in mentorship circles is beneficial. Just as Darwin's (2000) earlier work had shown, our focus groups learned that mentoring is about more than dispersing knowledge to mentees. We found that mentorship circles also offered a culture of support where mentees could comfortably share their experiences and learn from both mentors and mentees. Mentees noted that the hybridity of a themed and emergent approach to meeting structure was optimal. In a manner similar to Darwin and Palmer's (2009) findings, the discussion topics arose from group members and permitted mentees to prioritize their schedules. In addition we learned that, the emergent approach was crucial because it allowed the mentors to respond to mentees' pressing issues and dynamic needs. We found it was vital to structure time for open discussion so that mentees could explore more generally how they were doing and could share issues, ideas, and strategies.

A central finding that emerged was how the interdisciplinary nature of the mentorship circle facilitated mentees' capacity to navigate their role and foster collegiality. The interdisciplinary composition of mentorship circles was identified as a benefit, as it was by Darwin and Palmer (2009). Our evaluation elaborated how the interdisciplinary composition allowed mentees to draw upon diverse perspectives and identify disparities within the various schools. Additionally, the mentorship circle provided opportunities for mentees to build relationships across disciplines and schools, and create a sense of belonging and a broader sense of intellectual community within the university. Mentees indicated role expectations became clearer as they learned about each other's experiences across the various schools and shared their ideas and resources. 
Our evaluation results echo Jacobi's (1991) suggestion that mentorship relations have reciprocal benefits. The reciprocal nature of mentoring is more prominent in a group approach like a mentorship circle (Paludi, Martin, Stern, \& DeFour, 2010). As mentors in varying stages of our careers (including pre- and post-tenure), we found the experience of supporting and mentoring new colleagues rewarding. We also discovered that sharing our experiences in the group setting expanded our own understanding of the nuanced expectations of the faculty role and the university structure. Because of the varied stages of our careers, we were often learning alongside the mentees. Ultimately, the role of mentoring strengthened our collegiality and demonstrated a capacity for collaborative leadership.

Developing and offering the mentorship circle signalled that we value the importance of creating a space for new faculty members to be supported along their career paths. The mentees themselves indicated that the offering of a mentorship circle acts in ways to enhance the university's reputation and serves as a recruitment and retention strategy. The provision of a mentorship program demonstrates the university's interest and commitment to each faculty member. Currently, the mentorship circle is an initiative of only one of the six faculties at our university. As we found, it was important to have support from the dean or president's office in order to implement a mentorship program such as this.

Moving forward, we have identified a number of changes that would advance our mentorship circle model or assist others in implementing similar initiatives in their own settings. An important change would be to support further communication among mentees between the mentorship circle meetings. Although some mentees got together around shared activities, it was apparent that this was something that many others were interested in pursuing. Additionally, each cohort of the mentorship circle may continue to meet to provide ongoing support as they progress in their academic career. It might be worthwhile to organize an online forum restricted to mentorship circle members where follow-up and summaries of meetings can be posted and where discussions can continue in confidence.

\section{Conclusion}

On an individual level it seems evident that mentorship is a valuable strategy to support new faculty as they transition into their academic role. Mentorship models that align with the unique contexts of academic institutions have the potential to help new faculty members better understand the academic culture, establish collegial networks, experience a sense of belonging and support, develop a sense of confidence and commitment, and ultimately, to progress in their academic career. Of utmost importance is that mentorship circles provide faculty members with a sense of belonging and a safe space to discuss their concerns and learn strategies as they accommodate to their new role. The group format of a mentorship circle provides for the organic emergence of relationships where natural connections can emerge among mentees. We believe that mentoring new faculty members has the potential to enhance retention, foster greater commitment to the organization, increase research outcomes and publications rates, and contribute to the goals of the university. And finally, as mentors of the mentorship circle, we recognize that mentoring is an intellectual and relational journey, worthwhile for all participants. 


\section{Acknowledgements}

The authors would like to acknowledge Usha George, dean, Faculty of Community Services (FCS) for her unconditional support for the FCS Mentorship Circle Initiative. We would also like to express our appreciation to the FCS Mentorship Circle Members and focus group participants for their commitment to the success of this project. Thanks also go to Sonya Jancar for her contributions to the development of this manuscript and to Sarah Bukhari for the administrative support she provided to the mentorship circle facilitators.

\section{References}

Altbach, P. G. (Ed.). (2000). The changing academic workplace: Comparative perspectives. Chestnut Hill, MA: Boston College Center for International Higher Education.

Angelique, H., Kyle, K., \& Taylor, E. (2002). Mentors and muses: New strategies for academic success. Innovative Higher Education, 6(3), 195-209.

Beane-Katner, L. (2014). Anchoring a mentoring network in a new faculty development program. Mentoring \& Tutoring: Partnership in Learning, 22(2), 91-103. doi:10.1080/ 13611267.2014.902558

Belar, C. D. (1998). Graduate education in clinical psychology: "We're not in Kansas anymore." American Psychologist, 53(4), 456-464.

Blackwell, J. E. (1989). Mentoring: An action strategy for increasing minority faculty. Academe, 75(5), 8-14.

Chase, J., Topp, R., Smith, C., Cohen, M., Fahrenwald, N., Zerwic, J., \& Conn, V. (2013). Time management strategies for research productivity. Western Journal of Nursing Research, 35(2), 155-176.

Darwin, A. (2000). Critical reflections on mentoring in work settings. Adult Education Quarterly, 50, 197-211.

Darwin, A., \& Palmer, E. (2009). Mentoring circles in higher education. Higher Education Research \& Development, 28(2), 125-136.

Gazza, E. A. \& Shellenberger, T. (2005). Successful enculturation. Strategies for retaining newly hired nursing faculty. Nursing Educator. 30(6), 251-254.

Jacobi, M. (1991). Mentoring and undergraduate success: A literature review. Review of Educational Research, 61(4), 505-532.

Oosthuizen, P., Mckay, L., \& Sharpe, B. (2005). The problems faced by academics at various stages in their careers: The need for active institutional involvement. Retrieved from http://www.academia.edu/9176344/The_ProblemsFacedby_Academics_at_Various_ Stagesin_Their_Careers_The_Needfor_Active_Institutional_Involvement

Paludi, M., Martin, J., Stern, T., \& DeFour, D. (2010). Promises and pitfalls of mentoring women in business and academia. In C. Rayburn, F. Denmark, M. Reuder \& A. Austria (Eds.), A handbook for women mentors: Transcending barriers of stereotype, race and ethnicity (pp. 79-108). Santa Barbara, CA: Praeger. 
Rice, R., Sorcinelli, M., \& Austin, A. (2000). Heeding new voices: Academic careers for a new generation. Retrieved from http://files.eric.ed.gov/fulltext/ED451740.pdf

Savage, H., Karp, R., \& Logue, R. (2004). Faculty mentorship at colleges and universities. College Teaching, 52, 21-24.

Siler, B. B., \& Kleiner, C. (2001). Novice faculty: Encountering expectations in academia. Journal of Nursing Education, 40(9), 397-403.

White, A., Brannan, J., \& Wilson, C. B. (2010). A mentor-protégé program for new faculty, part I: Stories of protégés. Journal of Nursing Education, 49(11), 601-607.

Zeind, C.S., Zdanowicz, M., MacDonald, K., Parkhurst, C., King, C., \& Wizwer, P. (2005). Developing a sustainable faculty mentoring program. American Journal of Pharmaceutical Education, 65(5), 1-13.

\section{Contact Information}

Janice Waddell

Daphne Cockwell School of Nursing

Ryerson University

jwaddell@ryerson.ca

Jennifer Lapum is an associate professor at Ryerson University in the Daphne Cockwell School of Nursing. She has developed an arts-informed program of research with the aim of ensuring that no patient feels like "the 7,024th patient" when entering and exiting the health care system. She also employs arts media in her pedagogical approaches to advance experiential learning. She uses an array of arts media in her research and teaching including, but not limited to, installation art, poetry, images, dance, narrative, and music. Mentorship of students and new faculty is an important part of her scholarship.

Jennifer Martin is an associate professor in the School of Child and Youth Care at Ryerson University. Her program of research and scholarship focuses on trauma, child sexual abuse, child sexual abuse images online, child sexual exploitation, and cyber counselling. An integral component of her research entails collaboration with community agencies and organizations. Her research interests include the pedagogical role of mindfulness in teaching students in the helping professions. Student engagement and mentorship of new faculty are important components of her scholarship.

Jasna Schwind is an associate professor of nursing at Ryerson University. Her program of research, uses arts-informed narrative inquiry, which focuses on reconstruction of experience of personal and professional self within professional and therapeutic relationships in education and practice. To this end she developed Narrative Reflective Process (NRP), which is both a data collection tool as well as professional development instrument. This creative self-expression method includes storytelling, metaphors, drawing, creative writing and reflective dialogue. In her scholarship of teaching-learning, Jasna uses NRP to mentor and support students and peers. 
Janice Waddell is an associate dean in the Faculty of Community Services and an associate professor in the Daphne Cockwell School of Nursing, at Ryerson University. She has extensive teaching and curriculum development experience in undergraduate and graduate programs with particular foci on nursing education, leadership, and violence against women and children. Janice's research interests centre on the impact of curriculum-based career planning and development on student and new graduate nurse career resilience, faculty mentorship, student engagement, innovative curriculum design, and the transition experiences for undergraduate and graduate nursing students. 